Journal of Mathematics and Informatics

Vol. 12, 2018, 41-48

ISSN: 2349-0632 (P), 2349-0640 (online)

Published 28 February 2018

www.researchmathsci.org

DOI: http://dx.doi.org/10.22457/jmi.v12a5

Journal of

Mathematics and

Informatics

\title{
Dust Acoustic (DA) Waves in a Magnetised Plasma with Boltzmann Distributed Electrons and Ions
}

\author{
Sanchita Chetia \\ Department of Mathematics \\ Girijananda Choudhury Institute of Management and Technology \\ Azara, Guwahati-781017, Assam, India \\ E-mail: $\underline{\text { ndd48@ rediffmail.com }}$
}

Received 12 November 2017; accepted 2 January 2018

Abstract. The nonlinearity of dust-acoustic waves in a dusty plasma whose constituents are negatively charged dust, ions and electrons following Boltzmann distribution, is investigated by Sagdeev potential method. Existence of both subsonic and supersonic compressive solitons of interesting characters is established in this magnetized plasma model based on dust charge Zd (involved in f) and temperature ratio $\square$. The condition of the existence of solitons is

$$
\frac{k_{z}^{2}}{M^{2}}<\frac{1+(1-f) \alpha}{f}<\frac{1}{M^{2}}
$$

The amplitudes and depths of the potentials of subsonic and supersonic compressive solitons increase with the increase of dust charge Zd, but decrease with the increase of temperature ratio $\square$.

Keywords: Sagdeev Potential, Dust Acoustic Waves and Magnetized plasma.

AMS Mathematics Subject Classification (2010): 76E25

\section{Introduction}

The investigation of non-linear waves in various media is a present trend of research throughout the world. The particular study of the non-linear waves called solitary waves in plasma has attracted many researchers [1] towards its fold. This is a fascinating structure found in the most interesting space laboratory and even in terrestrial laboratory of the earth. Many investigations on solitary waves have already been made dealing with simple usual two component plasma, three component plasma and multi-component plasmas in magnetised and unmagnetised plasmas by various authors in international arena which are not reported here.

In the present trend of research, relativistic effects are being included in the ion, electron or beam components of various plasma models. The inclusion of relativistic effect is found to drastically change the property of solitary wave structures. Many workers such as Das and Paul [2], Nejoh [3], Das et al. [4], Pakira et al. [5], Kalita et al. [6], and Roychoudhury et al.[7] have investigated the existence of ion-acoustic solitary waves under various physical situations in relativistic plasmas. Lee and Choi [8] have 


\section{Sanchita Chetia}

investigated the ion-acoustic solitary wave in a relativistic plasma consisting of hot electrons and cold ions by Sagdeev pseudopotential method using a set of fully relativistic two-fluid equations. Very recently Kalita et al. [9] have investigated the existence of ion-acoustic relativistic solitons in an unmagnetised plasma with positive ion beam. In this investigation, they have considered lower and higher order relativistic effects. Recently Kalita and Deka (2013) [1] have investigated ion acoustic solitons in a weakly relavistic magnetised plasma based on initial steaming speeds in which the lower limit of the speed of light to characterise relativistic compressive solitons is predicted. Besides, Kalita and Choudhury (2013) [10] have also established the role of unidirected relativistic electrons with inertia in the formation of the weakly relativistic ion acoustic solitons in magnetised plasma. Both subsonic and supersonic compressive solitons are established. The generation of compressive solitons are shown to confine near the vicinity of the direction of the magnetic field in small subsonic range.

Besides, the presence of dust components in space plasma has changed the attitude of researchers to investigate its effect in the formation of solitary waves. Rao et al. [11] have studied the existence of solitary waves in a simple model with cold dust components with Boltzmann distributed electrons and ions. Shukla and Mamun [12] have described the various effects of dust charges in space plasma. Verheest and Pillay [13] have studied solitary waves in a dusty plasma with Cairns distributed ions and Boltzmann electrons. He has reported the effect of the parameter $\square$ based on a non-thermal parameter $\square$ where $=\frac{4 \alpha}{1+3 \alpha}$. Besides DA and DIA solitons in space plasma with stationary or mobile dusts have been investigated to conclude solitons of positive or negative potentials on the basis of implicitly occurring dust charges $Z_{d}$ in some forms. But relativistic effects to the small particles electrons and ions in the space regions, like Van Allen Radiation belt, laser plasma interaction, earth's magnetosphere, outer boundary of radiation belt etc are not considered when dust charges may be present. DIA solitons in an unmagnetized plasma with relativistic ions, Boltzmann electrons (where relativistic effects is not explicit) and stationary dusts are discussed only by Liu et al. [14, 15, 16].

Recently quantum effects Mamun et al. [17], Hass and Manfrendi [18], Hass et al. [19] are adopted to change the characteristic behaviour of solitary waves in plasma. Further Hass [20], Misra and Chowdhury [21] and Mahmood [22] have studied solitary waves in magnetised and unmagnetised plasmas with the inclusion of quantum effects.

In this paper, we have considered magnetised dusty plasma supplemented by Boltzmann distributed electrons and ions. The energy integral in this magnetised dusty plasma is established to establish the existence of solitary waves.

\section{Equations governing the dynamics of motion}

In order to establish the existence of solitary waves in a magnetized dusty plasma, we start with a set of interpenetrating fluid characterized by the equations of continuity and motion of the negatively charged dust particles together with Boltzmann distributed electrons and ions. The governing equations are as follows:

$$
\begin{aligned}
& \frac{\partial n_{d}}{\partial t}+\frac{\partial}{\partial x}\left(n_{d} v_{d x}\right)+\frac{\partial}{\partial z}\left(n_{d} v_{d z}\right)=0 \\
& \frac{\partial v_{d x}}{\partial t}+v_{d x} \frac{\partial v_{d x}}{\partial x}+v_{d z} \frac{\partial v_{d x}}{\partial z}=\frac{\partial \varphi}{\partial x}+v_{d y}
\end{aligned}
$$


Dust Acoustic (DA) Waves in a Magnetised Plasma with Boltzmann Distributed Electrons and Ions

$$
\begin{aligned}
& \frac{\partial v_{d y}}{\partial t}+v_{d x} \frac{\partial v_{d y}}{\partial x}+v_{d z} \frac{\partial v_{d y}}{\partial z}=-v_{d x} \\
& \frac{\partial v_{d z}}{\partial t}+v_{d x} \frac{\partial v_{d z}}{\partial x}+v_{d z} \frac{\partial v_{d z}}{\partial z}=\frac{\partial \varphi}{\partial z} \\
& n_{e}=(1-f) e^{\alpha \varphi} \\
& n_{i}=e^{-\varphi}
\end{aligned}
$$

We have normalised densities by $n_{i 0}$, velocities by $C_{d}=\left(k Z_{d} / m_{d}\right)^{1 / 2}$, potential by $\left(k T_{i}\right) / e$, time by gyro-period $\Omega_{\mathrm{d}}{ }^{-1}=\frac{\mathrm{m}_{\mathrm{d}}}{\mathrm{B}_{0} \mathrm{Z}_{\mathrm{d}} \mathrm{e}}$ and distances by gyroradius $C_{d} \Omega_{\mathrm{d}}{ }^{-1}, k$ being the Boltzmann constant.

$$
\text { Here } f=\frac{Z_{d} n_{d 0}}{n_{i 0}} \text { and } \alpha=\frac{T_{i}}{T_{e}}
$$

\section{Derivation of energy integral}

We assume that the wave is propagating obliquely to the external magnetic field depending on the quantity

$$
\begin{aligned}
& \xi=x k_{x}+z k_{z}-M t \\
& \text { where } k_{x}{ }^{2}+k_{z}{ }^{2}=1 \text { and } M=\frac{V}{C_{d}} \\
& \text { then } k_{x} \frac{\partial}{\partial \xi}=\frac{\partial}{\partial x}, k_{z} \frac{\partial}{\partial \xi}=\frac{\partial}{\partial z},-M \frac{\partial}{\partial \xi}=\frac{\partial}{\partial t} \\
& \text { using this transformation in (1)-(4), we get } \\
& k_{x} v_{d x}+k_{z} v_{d z}=M\left(1-\frac{f}{Z_{d} n_{d}}\right) \\
& \quad-\frac{M f}{Z_{d} n_{d}} \frac{\partial v_{d x}}{\partial \xi}=k_{x} \frac{\partial \varphi}{\partial \xi}+v_{d y} \\
& \quad \frac{M f}{Z_{d} n_{d}} \frac{\partial v_{d y}}{\partial \xi}=v_{d x} \\
& \frac{M f}{Z_{d} n_{d}} \frac{\partial v_{d z}}{\partial \xi}=k_{z} \frac{\partial \varphi}{\partial \xi}
\end{aligned}
$$$$
\text { using this transformation in (1)-(4), we get }
$$

From (8), (9) and (11) we get

$$
-\frac{M^{2} f^{2}}{Z_{d}^{2}} \frac{1}{n_{d}{ }^{3}} \frac{\partial n_{d}}{\partial \xi}-\frac{\partial \varphi}{\partial \xi}=k_{x} v_{d y}
$$

Using charge neutrality condition, we get,

$$
\begin{aligned}
& Z_{d} n_{d}+n_{e}=n_{i} \\
& \Rightarrow n_{d}=\frac{1}{z_{d}}\left\{e^{-\varphi}-(1-f) e^{\alpha \varphi}\right\}
\end{aligned}
$$

From (11) and (13) we get,

$$
v_{d z}=\frac{k_{z}}{M f}\left(e^{-\varphi}+\frac{1-f}{\alpha} e^{\alpha \varphi}-1-\frac{1-f}{\alpha}\right)
$$

From (8) we get,

$$
k_{x} v_{d x}=M\left\{1-\frac{f}{e^{-\varphi}-(1-f) e^{\alpha \varphi}}\right\}-\frac{k_{z}^{2}}{M f}\left(e^{-\varphi}+\frac{1-f}{\alpha} e^{\alpha \varphi}-1-\frac{1-f}{\alpha}\right)
$$

From (10) we get,

$$
\frac{M f}{Z_{d} n_{d}} \frac{\partial}{\partial \xi}\left(k_{x} v_{d y}\right)=k_{x} v_{d x}
$$

From (12), (13), (15) and (16) we get,

$$
\begin{gathered}
\frac{1}{2} \frac{\partial}{\partial \xi}\left[\left\{1-M^{2} f^{2} \frac{e^{-\varphi}+(1-f) \alpha e^{\alpha \varphi}}{\left\{e^{-\varphi}-(1-f) e^{\alpha \varphi}\right\}^{3}}\right\}^{2}\left(\frac{\partial \varphi}{\partial \xi}\right)^{2}\right]=\left[-\frac{1}{f}\left\{e^{-\varphi}-(1-f) e^{\alpha \varphi}\right\}+1+\right. \\
M^{2} f \frac{e^{-\varphi}+(1-f) \alpha e^{\alpha \varphi}}{\left\{e^{-\varphi}-(1-f) e^{\alpha \varphi}\right\}^{2}}-M^{2} f^{2} \frac{e^{-\varphi}+(1-f) \alpha e^{\alpha \varphi}}{\left\{e^{-\varphi}-(1-f) e^{\alpha \varphi}\right\}^{3}}+\frac{k_{z}{ }^{2}}{M^{2} f^{2}}\left\{e^{-\varphi}-(1-f) e^{\alpha \varphi}\right\}\left(e^{-\varphi}+\right.
\end{gathered}
$$




\section{Sanchita Chetia}

$\left.\left.\frac{1-f}{\alpha} e^{\alpha \varphi}-1-\frac{1-f}{\alpha}\right)-k_{z}{ }^{2} \frac{e^{-\varphi}+(1-f) \alpha e^{\alpha \varphi}}{\left\{e^{-\varphi}-(1-f) e^{\alpha \varphi}\right\}^{2}}\left(e^{-\varphi}+\frac{1-f}{\alpha} e^{\alpha \varphi}-1-\frac{1-f}{\alpha}\right)\right] \frac{\partial \varphi}{\partial \xi}$

Integrating we get,

$$
\begin{aligned}
& \frac{1}{2}\left(\frac{d \varphi}{d \xi}\right)^{2}=\frac{1}{\left\{1-M^{2} f^{2} \frac{e^{-\varphi}+(1-f) \alpha e^{\alpha \varphi}}{\left\{e^{-\varphi}-(1-f) e^{\alpha \varphi}\right\}^{3}}\right\}^{2}}\left[\frac{1}{f}\left(e^{-\varphi}+\frac{1-f}{\alpha} e^{\alpha \varphi}-1-\frac{1-f}{\alpha}\right)+\varphi+\right. \\
& \left\{\frac{1}{e^{-\varphi}-(1-f) e^{\alpha \varphi}}-\frac{1}{f}\right\}-\frac{M^{2} f^{2}}{2}\left\{\frac{1}{\left\{e^{-\varphi}-(1-f) e^{\alpha \varphi}\right\}^{2}}-\frac{1}{f^{2}}\right\}-\frac{k_{z}{ }^{2}}{2 M^{2} f^{2}}\left(e^{-\varphi}+\frac{1-f}{\alpha} e^{\alpha \varphi}-1-\right. \\
& \left.\frac{1-f}{\alpha}\right)^{2}-k_{z}{ }^{2} \frac{\left(e^{-\varphi}+\frac{1-f}{\alpha} e^{\alpha \varphi}-1-\frac{1-f}{\alpha}\right)}{e^{-\varphi}-(1-f) e^{\alpha \varphi}}-k_{Z}{ }^{2} \varphi \\
& \text { which can be written as } \\
& \frac{1}{2}\left(\frac{d \varphi}{d \xi}\right)^{2}+K\left(\varphi, \alpha, M, k_{z}, f\right)=0
\end{aligned}
$$

$K(\varphi)$ is the Sagdeev potential $K(\varphi)$, a function of potential $\varphi$ with variable parameters $\square \square \mathrm{M}, \mathrm{k}_{\mathrm{z}}$ and $\mathrm{f}$. given by

For maximum variation $\varphi_{m}$ (amplitude) of the non linear dispersion equation is

$$
K\left(\varphi_{m}\right)=0
$$

This determines the amplitude $\varphi_{m}$ of soliton for a given set of parameters.

\section{Conditions for the existence of solitary waves}

Appropriate and necessary conditions for the existence of solitary wave solitons can be obtained by studying the behavior of the potential $K(\varphi)$ near $\varphi=0$ and $\varphi=\varphi_{m}$. For solitary wave solitons, the conditions required are

$$
\begin{aligned}
& K(0)=K\left(\varphi_{m}\right)=K^{\prime}(0)=0 \\
& \text { and } \\
& K(\varphi)<0 \text {, between } \varphi=0 \text { and } \varphi=\varphi_{m}
\end{aligned}
$$

To ascertain the values of amplitudes and the shape of the potential between the points $\varphi=0$ and $=\varphi_{m}$, it is desirable to investigate the behavior of the potential $K(\varphi)$ near its' zeros. Expanding $K(\varphi)$, by Taylor, s expansion, near $\varphi \approx 0$ and $\varphi \approx \varphi_{m}$, we obtain

$$
\begin{gathered}
K(\varphi \approx 0)=-\frac{\varphi^{2}}{2} \frac{\left\{\frac{1}{f}\{1+(1-f) \alpha\}-\frac{k_{z}{ }^{2}}{M^{2}}\right\}}{\left\{1-\frac{M^{2}}{f}\{1+(1-f) \alpha\}\right\}} \\
K\left(\varphi \approx \varphi_{m}\right)=-\frac{\varphi-\varphi_{m}}{1-M^{2} f^{2} \frac{e^{-\varphi_{+}(1-f) \alpha e^{\alpha \varphi}}}{\left\{e^{\left.-\varphi_{-}(1-f) e^{\alpha \varphi}\right\}^{3}}\right.}\left[1-\frac{1}{f}\left\{e^{-\varphi_{m}}-(1-f) e^{\alpha \varphi_{m}}\right\}+\right.} \\
\left.\frac{k_{z}^{2}}{M^{2} f^{2}}\left(e^{-\varphi_{m}}+\frac{1-f}{\alpha} e^{\alpha \varphi_{m}}-1-\frac{1-f}{\alpha}\right)\left\{e^{-\varphi_{m}}-(1-f) e^{\alpha \varphi_{m}}\right\}\right]
\end{gathered}
$$

exist subject to

From (23) and (24) under condition (22), we observe that solitons

$$
\frac{1}{f}\{1+(1-f) \alpha\}>\frac{k_{z}^{2}}{M^{2}}>\frac{k_{z}^{2}}{f}\{1+(1-f) \alpha\}
$$


Dust Acoustic (DA) Waves in a Magnetised Plasma with Boltzmann Distributed Electrons and Ions

\section{Conclusion and discussion}

In this model of magnetised dusty plasma in presence of Boltzmann distributed electrons and ions, solitary waves of interesting characters are shown to exist.

Existence of both subsonic compressive and rarefactive solitons is established in this dusty plasma with mobile negative dust charges associated with Boltzmann distributed ions and electrons. The amplitudes of the compressive DA solitons are found to be much larger than those of rarefactive solitons (Fig-1) for the same set of plasma parameters. Further, the higher potential depths of compressive DA solitons could accommodate greater amount of plasma particles (dense) but the corresponding counterpart of rarefactive solitons could contain lesser number of particles characterised by smaller potential depth.

The amplitudes of subsonic compressive and rarefactive DA solitons are found to be increased [Fig-2] when the direction of propagation is nearly in the perpendicular direction to the magnetic field. Otherwise in the magnetised dusty plasma, high amplitude subsonic compressive and rarefactice solitons are dependent on the direction of propagation and small $\mathrm{Z}_{\mathrm{d}}$. The characteristic behaviour of the magnitude of soliton amplitude is reflected in figure- 3 when $f(=0.05)$ becomes still smaller for small $Z_{d}$ supplemented by small $\mathrm{n}_{\mathrm{d} 0}$. For this change, the amplitudes of solitons are found to be considerably reduced.

To the contrary, the amplitudes of the subsonic compressive and rarefactive DA solitons are seen to increase substantiably at the increase of $f(=0.4)$ i.e. at the increase of dust charge $Z_{d}$ and $n_{d 0}$ suplemented by the mach number $M(=0.5)$. Deposition of more dust charges with faster speed (M) appears to produce high amplitude solitons through much larger potential depths [Fig-4].

\section{REFERENCES}

1. B.C.Kalita and M.Deka, Investigation of ion acoustic solitons (ias) in a weakly relativistic magnetized plasma astrophys, Space Sci., 347 (2013) 109-118.

2. G.C.Das and S.N.Paul, Ion-acoustic solitary waves in relativistic plasma, Phys. Fluids, 28 (1985) 823-834.

3. Y.Nejoh, The effect of the ion temperature on the ion acoustic solitary waves in a collisionless relativistic plasma, J. Plasma Phys., 37 (1987) 487-498.

4. G.C.Das, B.Karmakar, and S.N.Paul, Propagation of solitary waves in relativistic plasmas, IEEE Trans. Plasma Sci., 16 (1988) 22-29.

5. G.Pakira, A.Roychowdhury and S.N.Paul, Higher order corrections to the ionacoustic waves in a relativistic plasma (isothermal case), J. Plasma Phys., 40 (1988) 359-368.

6. B.C.Kalita, S.N.Barman and G.Goswami, Weakly relativistic solitons in a cold plasma with electron inertia, Phys. Plasmas, 3 (1996) 145-148.

7. R.Roychoudhury, S.K.Venkatesan, and C.Das, Effects of ion and electron drifts on large amplitude solitary waves in a relativistic plasma, Phys. Plasmas, 4 (1997) 42324237.

8. N.Lee and C.Choi, On-acoustic solitary waves in a relativistic plasma, Phys. Plasmas, 14 (2007) 022307-022311. 


\section{Sanchita Chetia}

9. B.C.Kalita, R.Das, and H.K.Sarmah, Weakly relativistic effect in the formation of ion-acoustic solitary waves in a positive ion-beam plasma, Can. J. Phys., 88 (2010) 157-163.

10. B.C.Kalita and M.Choudhury, The role of undirected relativistic electrons with inertia in the formation of weakly relativistic ion acoustic solitons in magnetized plasma, Astrophys. Space Sci., 346 (2013) 375-383.

11. N.N.Rao, P.K.Shukla, M.Y.Yu, Dust-acoustic waves in dusty plasmas planet, Space Sci., 38 (1990) 543-549.

12. P.K.Shukla and A.A.Mamun, Introduction to Dusty Plasma Phys. (IOP, Bristol, London, 2002) 45-52.

13. F.Verheest, and S.R.Pillay, Dust-acoustic solitary structures in plasmas with nonthermal electrons and positive dust, Phys. Plasmas, 15 (2008) 013703 (2008) 203-211.

14. B.C.Kalita and S.Das, Dust ion acoustic (DIA) solitary waves in plasmas with weak relativistic effects in electrons and ions, Astrophys. Space Sci., 352 (2014) 585-593.

15. H.F.Liu, S.Q.Wang, C.Z.Li, Q.Xiang, F.Z.Yang, and Y.Liu, Cylindrical and spherical dust-ion acoustic solitary waves in a relativistic dust plasm, Physica Scripta, 82 (2010) 065402-065407.

16. H.F.Liu, S.Q.Wang, Z.Wang, F.Z.Yang, Y.Liu, and S.Li, Advances in Space Res., 51 (2013) 2368-2375.

17. A.A.Mamun, R.A.Cairns, P.K.Shukla, Effects of vortex-like and nonthermal ion distributions on non-linear dust-acoustic waves, Phys. Plasmas, 3 (1996) 2610-2617.

18. G.Manfrendi and F.Hass, Self-consistent fluid model for a quantum electron gas, Phys. Rev. B, 64 (2001) 075316-075320.

19. F.Hass, L.G.Garcia, J.Goedest and G.Manfrendi, Quantum ion-acoustic waves, Phys. Plasma, 10 (2003) 3858-3865.

20. F.Hass, A Magnetohydrodynamic model for quantum plasmas, Phys. Plasma, 12 (2005) 062117-062127.

21. A.P.Misra and A.R.Chowdhury, Modulation of dust acoustic waves with a quantum correction, Physics of Plasma, 13 (2006) 0723075-0723082.

22. S.Mahmood, Arbitrary amplitude dust ion acoustic solitary waves in dense fermi plasmas, Physics of Plasma, 15 (2008) 014502-014511.

23. B.C.Kalita and S.Das, Dust ion acoustic (DIA) solitary waves in plasmas with weak relativistic effects in electrons and ions, Astrphys. Spce Sci. Astrophys. Space Sci., 352 (2014) 585-594.

24. B.C.Kalita and R.Kalita, A New Approach to Energy Integral for Investigation of Dust- Ion Acoustic (DIA) Waves in Multi-Component Plasmas with Quantum Effects in Inertia Less Electrons,Communications in Theoritical Physics, 63 (2015) 67-75. 
Dust Acoustic (DA) Waves in a Magnetised Plasma with Boltzmann Distributed Electrons and Ions

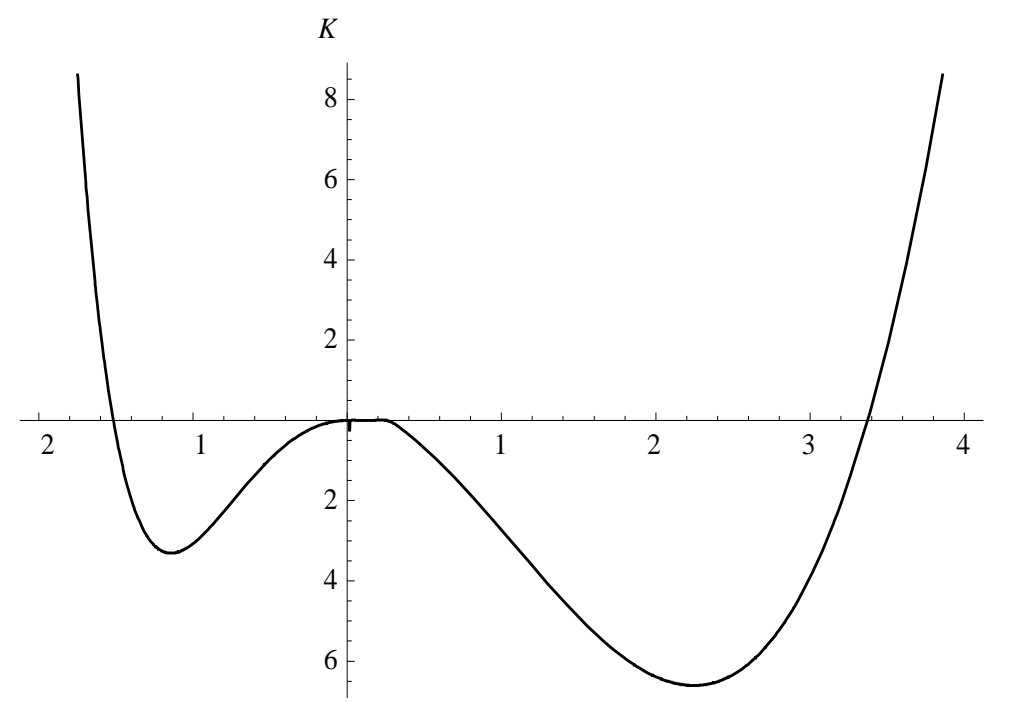

Figure 1: Sagdeev potentials to characterise both compressive and rarefactive solitons for $\mathrm{k}_{\mathrm{z}}=0.1, \mathrm{M}=0.3, \square=0.2$ and $\mathrm{f}\left(=Z_{\boldsymbol{d}} \frac{\boldsymbol{n}_{\boldsymbol{d} \mathbf{0}}}{\boldsymbol{n}_{\boldsymbol{i} \mathbf{0}}}\right)=0.15$

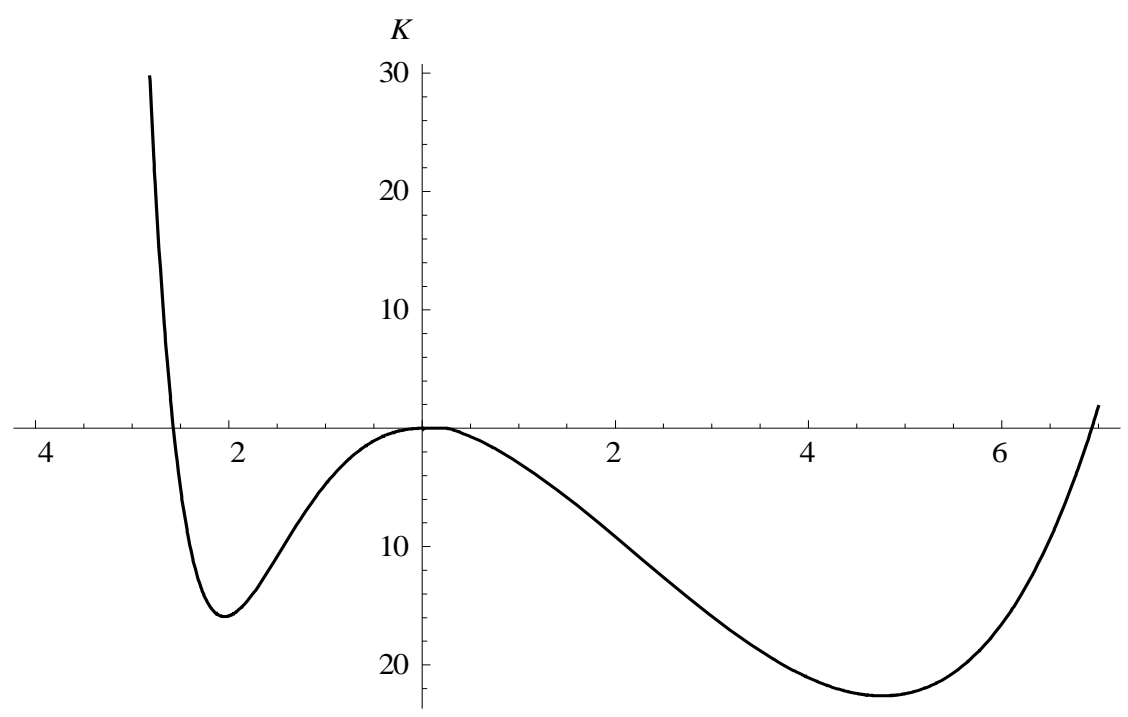

Figure 2: Sagdeev potentials to characterise both compressive and rarefactive solitons for $\mathrm{k}_{\mathrm{z}}=0.05, \mathrm{M}=0.3, \square=0.2$ and $\mathrm{f}=0.15$ 


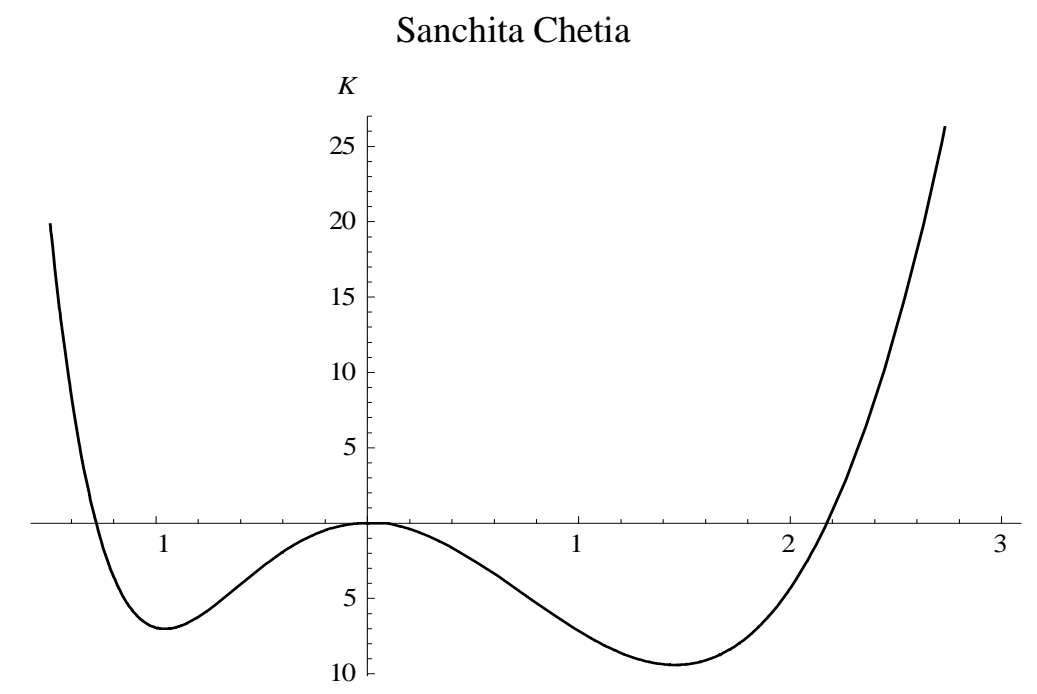

Figure 3: Sagdeev potentials to characterise both compressive and rarefactive solitons for $\mathrm{k}_{\mathrm{z}}=0.05, \mathrm{M}=0.2, \square=0.2$ and $\mathrm{f}=0.05$

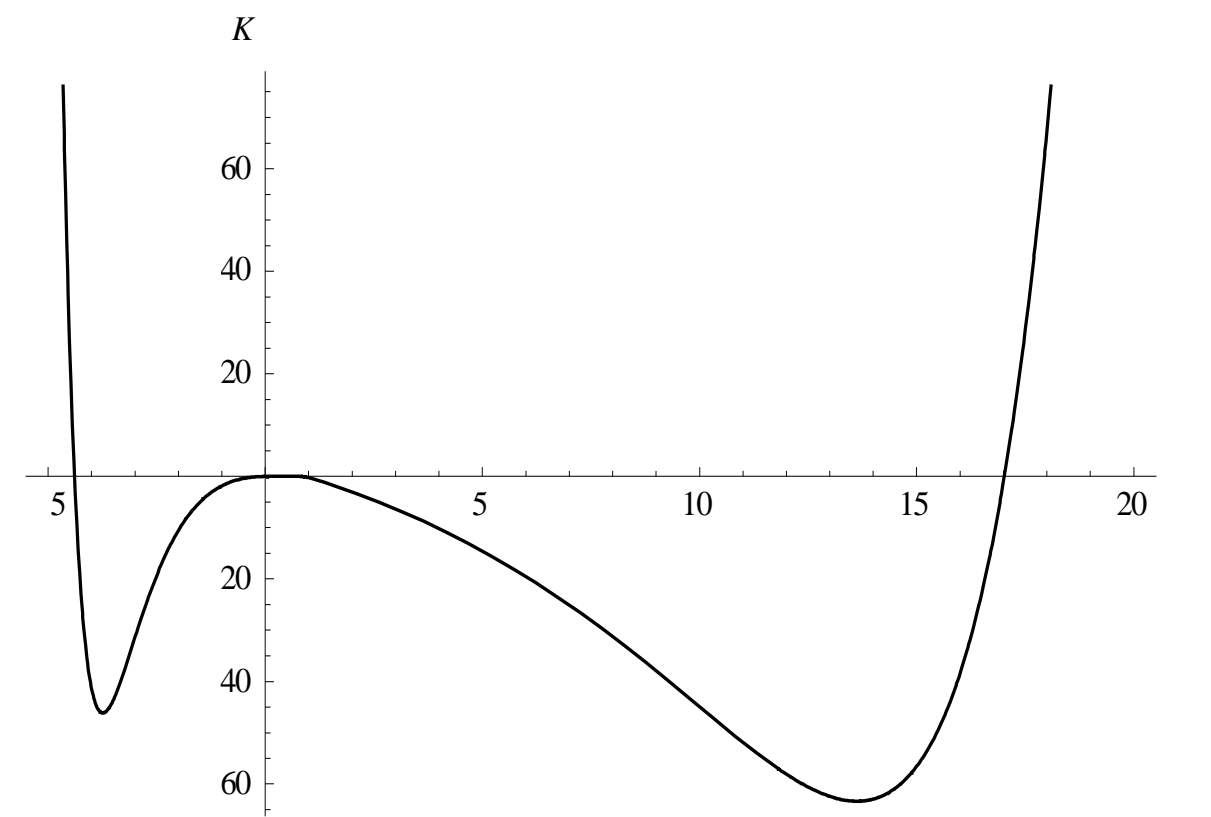

Figure 4: Sagdeev potentials to characterise both compressive and rarefactive solitons for $\mathrm{k}_{\mathrm{z}}=0.05, \mathrm{M}=0.5, \square=0.2$ and $\mathrm{f}=0.4$ 